PROCEEDINGS OF THE

AMERICAN MATHEMATICAL SOCIETY

Volume 131, Number 3, Pages 741-744

S 0002-9939(02)06802-8

Article electronically published on October 15, 2002

\title{
GAUSSIAN CURVATURE IN THE NEGATIVE CASE
}

\author{
WENXIONG CHEN AND CONGMING LI
}

(Communicated by Bennett Chow)

\begin{abstract}
In this paper, we reinvestigate an old problem of prescribing Gaussian curvature in the negative case.

In 1974, Kazdan and Warner considered the equation

$$
-\triangle u+\alpha=R(x) e^{u}, \quad x \in M,
$$

on any compact two dimensional manifold $M$ with $\alpha<0$. They showed that there exists a number $\alpha_{o}$, such that the equation is solvable for every $0>\alpha>\alpha_{o}$ and it is not solvable for $\alpha<\alpha_{o}$.

Then one may naturally ask:

Is the equation solvable for $\alpha=\alpha_{o}$ ?

In this paper, we answer the question affirmatively. We show that there exists at least one solution for $\alpha=\alpha_{o}$.
\end{abstract}

\section{INTRODUCTION}

Prescribing Gaussian curvature is an interesting problem in Riemannian geometry. Given a function $K(x)$ on a two dimensional manifold $M$ with metric $g_{o}$, can it be realized as the Guassian curvature of some metric $g$ which is pointwise conformal to $g_{o}$ ? This is equivalent to the solvability of the semilinear elliptic equation

$$
-\triangle u+k_{o}(x)=K(x) e^{2 u}
$$

where $\triangle$ is the Laplace operator and $k_{o}(x)$ the Gaussian curvature associated with the metric $g_{0}$.

By making a proper change of variables, one can easily arrive at the following simpler equation:

$$
-\triangle u+\alpha=R(x) e^{u}, \quad x \in M .
$$

In [6], Kazdan and Warner considered this equation on any compact two dimensional manifold $M$ with $\alpha<0$. This corresponds to prescribing Gaussian curvature on negatively curved manifolds.

Let $\bar{R}$ be the average of $R$. Kandan and Warner showed that

i) A necessary condition for (11) to have a solution is $\bar{R}<0$.

ii) If $\bar{R}<0$, then there is a constant $-\infty \leq \alpha_{o}<0$, such that one can solve (11) if $\alpha_{o}<\alpha$, but cannot solve (1) if $\alpha<\alpha_{o}$.

iii) $\alpha_{o}=-\infty$ if and only if $R \leq 0$.

Received by the editors October 12, 2000.

2000 Mathematics Subject Classification. Primary 35J60.

The first author was partially supported by NSF Grant DMS-0072328.

The second author was partially supported by NSF Grant DMS-9970530.

(C)2002 American Mathematical Society 
One can see from their results that in the case when $R$ is nonpositive, the problem has been solved completely. However in the case when $R$ changes signs, one has $\alpha_{o}>-\infty$, hence there are some remaining questions. A natural one is:

Can one solve (1) when $\alpha=\alpha_{o}$ ?

The main purpose of this paper is to answer the above question. We prove

Theorem 1. Let $\alpha_{o}>-\infty$ be defined above. Then equation (1) has at least one solution when $\alpha=\alpha_{o}$.

We will prove the theorem in the next section. The main idea is the following.

Let $\alpha_{k}$ be a sequence of numbers such that

$$
\alpha_{k}>\alpha_{o}, \text { and } \alpha_{k} \rightarrow \alpha_{o} \text {, as } k \rightarrow \infty \text {. }
$$

For each $\alpha_{k}$, we minimize the functional

$$
J_{k}(u)=\frac{1}{2} \int_{M}|\nabla u|^{2}+\alpha_{k} \int_{M} u-\int_{M} R(x) e^{u}
$$

in a class of functions that are between a sub and a super solution. Let the minimizer be $u_{k}$. We show that the sequence $\left\{u_{k}\right\}$ so chosen is bounded and converges to a solution corresponding to $\alpha=\alpha_{o}$.

\section{The Proof of Theorem 1}

In this section, we prove the existence of a solution for $\alpha=\alpha_{o}$. The proof is divided into three steps.

In Step 1, we minimize the functional $J_{k}(\cdot)$ for each $\alpha_{k}>\alpha_{o}$. Then let $\alpha_{k} \rightarrow \alpha_{o}$.

In Step 2, we show that the sequence of minimizers $\left\{u_{k}\right\}$ is bounded in the region where $R(x)$ is positively bounded away from 0 .

In Step 3, we prove that $\left\{u_{k}\right\}$ is bounded in $H^{1}(M)$ and hence converges to a desired solution.

Step 1. Let $\left\{\alpha_{k}\right\}$ be a sequence of numbers such that

$$
0>\alpha_{k}>\alpha_{o} \text {, and } \alpha_{k} \rightarrow \alpha_{o} \text {. }
$$

Consider the functional

$$
J_{k}(u)=\frac{1}{2} \int_{M}|\nabla u|^{2}+\alpha_{k} \int_{M} u-\int_{M} R(x) e^{u}
$$

in Sobolev space $H^{1}(M)$.

Choose a sufficiently negative constant $A$ to be the subsolution of equation (1) for all $\alpha_{k}$, that is,

$$
-\triangle A+\alpha_{k} \leq R(x) e^{A}
$$

This is obviously possible.

For each fixed $\alpha_{k}$, choose $\alpha_{o}<\tilde{\alpha}_{k}<\alpha_{k}$. By the result of Kazdan and Warner 6], there exists a solution of equation (11) for $\alpha=\tilde{\alpha}_{k}$. Call it $\psi_{k}$. Obviously, $\psi_{k}$ is a super solution for equation

$$
-\triangle u+\alpha_{k}=R(x) e^{u}
$$

Illuminated by an idea of Ding and Liu [4], we minimize the functional $J_{k}(\cdot)$ in the class of functions

$$
H=\left\{u \in C^{1}(M) \mid A \leq u \leq \psi_{k}\right\}
$$


Through some standard variational argument (for example, see [4] or [2]), one can conclude that a minimizer $u_{k}$ of $J_{k}(\cdot)$ exists and is in the interior of $H$. Obviously, $u_{k}$ is the solution of equation (11) for $\alpha=\alpha_{k}$.

Step 2. Since the sequence $\left\{u_{k}\right\}$ is uniformly bounded from below, to show that it is also bounded from above in the region where $R(x)$ is positively bounded away from 0, we use a result of Brezis and Li [1].

Lemma 2.1. Assume that $V$ is a Lipschitz function satisfying

$$
0<a \leq V(x) \leq b<\infty
$$

and let $K$ be a compact subset of a domain $\Omega$ in $R^{2}$. Then any solution $u$ of

$$
-\triangle u=V(x) e^{u}, \quad x \in \Omega,
$$

satisfies

$$
\sup _{K} u+\inf _{K} u \leq C\left(a, b,\|\nabla V\|_{L^{\infty}}, K, \Omega\right) .
$$

In fact, we [3] recently improved their result so that the constant $C$ is independent of $b$ - the upper bound of $R(x)$.

Let $x_{o}$ be a point at which $R\left(x_{o}\right)>0$. Let $\epsilon$ be so small such that

$$
R(x)>0 \quad \forall x \in B_{\epsilon}\left(x_{o}\right) .
$$

Let $\psi_{k}$ be a solution of

$$
\begin{cases}-\triangle \psi_{k}-\alpha_{k}=0 & x \in B_{\epsilon}\left(x_{o}\right) \\ \psi_{k}(x)=1 & x \in \partial B_{\epsilon}\left(x_{o}\right) .\end{cases}
$$

Let $w_{k}=u_{k}+\psi_{k}$. Then $w_{k}$ satisfies

$$
-\triangle w_{k}=R(x) e^{-\psi_{k}} e^{w_{k}} .
$$

It is obvious that the sequence $\left\{\psi_{k}\right\}$ is bounded from above and below in the small ball. Since $\left\{u_{k}\right\}$ is uniformly bounded from below, $\left\{w_{k}\right\}$ is also bounded from below. Locally, the metric is pointwise conformal to the Euclidean metric, so one can apply Lemma 2.1 to $\left\{w_{k}\right\}$ and conclude that the sequence $\left\{w_{k}\right\}$ is also bounded from above in the small ball, and hence bounded in the region where $R(x)$ is positively bounded away from zero. Now the bound for $\left\{u_{k}\right\}$ follows suit.

Step 3. We show that the sequence $\left\{u_{k}\right\}$ is bounded in $H^{1}(M)$.

On one hand, since $u_{k}$ is a minimizer of the functional $J_{k}$, the second derivative $J_{k}^{\prime \prime}\left(u_{k}\right)$ is positively definite. It follows that for any function $\phi$,

$$
\int_{M}\left(|\nabla \phi|^{2}-R(x) e^{u_{k}} \phi^{2}\right) \geq 0 .
$$

Choosing $\phi=e^{\frac{u_{k}}{2}}$, we have

$$
\frac{1}{4} \int_{M}\left|\nabla u_{k}\right|^{2} e^{u_{k}} \geq \int_{M} R(x) e^{2 u_{k}} .
$$

On the other hand, multiplying both sides of the equation

$$
-\triangle u_{k}+\alpha_{k}=R(x) e^{u_{k}}
$$

by $e^{u_{k}}$ and integrating, we have

$$
\int_{M}\left(\left|\nabla u_{k}\right|^{2} e^{u_{k}}+\alpha_{k} e^{u_{k}}\right)=\int_{M} R(x) e^{2 u_{k}} .
$$


Combining (4) and (5), we arrive at

$$
-\alpha_{k} \int_{M} e^{u_{k}} \geq \frac{3}{4} \int_{M}\left|\nabla u_{k}\right|^{2} e^{u_{k}} .
$$

If we let $v_{k}=e^{\frac{u_{k}}{2}}$, then (6) becomes

$$
\int_{M}\left|\nabla v_{k}\right|^{2} \leq C \int_{M} v_{k}^{2}
$$

for some positive constant $C$.

In Step 2, we have shown that $\left\{u_{k}\right\}$ is bounded in the region where $R(x)$ is bounded away from 0 , as does $\left\{v_{k}\right\}$ there. It follows from (7) that $\left\{v_{k}\right\}$ is bounded in $H^{1}(M)$, therefore, $\left\{u_{k}\right\}$ is also bounded in $H^{1}(M)$. Consequently, there exists a subsequence of $\left\{u_{k}\right\}$ that converges to a function $u_{o}$ in $H^{1}(M)$, which is the desired solution of equation (11) for $\alpha=\alpha_{o}$.

This completes the proof of the Theorem.

\section{REFERENCES}

1. H.Brezis, Y.Y.Li, A sup+inf inequality for some nonlinear elliptic equations involving exponential nonlinearities, J. Funct. Analysis, 115(1993) 344-358. MR 94g:35080

2. K.C.Chang, Infinite dimensional Morse theory and multiple solution problems, Birkhauser, 1993. MR 94e:58023

3. W.Chen, C.Li, Moving planes, moving spheres, and apriori estimates, preprint, 2000

4. W.Ding, J.Liu, A note on the problem of prescribing Gaussian curvature on surfaces, Trans. AMS, 347(1995) 1059-1065. MR 95e:53058

5. D.Gilbarg, N.S.Trudinger, Elliptic partial differential equations of second order, SpringerVerlag, 1983. MR 86c:35035

6. J.Kazdan, F. Warner, Curvature functions for compact two manifolds, Ann. of Math., 99(1974) 14-47. MR 49:7949

Department of Mathematics, Southwest Missouri State University, Springfield, MisSOURI 65804

E-mail address: wec344f@smsu.edu

Department of Applied Mathematics, University of Colorado at Boulder, Boulder, Colorado 80039

E-mail address: cli@newton.colorado.edu 